\title{
Temporal mental qualities and selective attention
}

\author{
Michał Klincewicz \\ Department of Cognitive Science \\ Jagiellonian University in Krakow \\ michal.klincewicz@gmail.com
}

\begin{abstract}
This article presents an argument for the view that we can perceive temporal features without awareness. Evidence for this claim comes from recent empirical work on selective visual attention. An interpretation of selective attention as a mechanism that processes high-level perceptual features is offered and defended against one particular objection. In conclusion, time perception likely has an unconscious dimension and temporal mental qualities can be instantiated without ever being conscious.
\end{abstract}

Keywords: time perception; conscious experience of time; N2pc; perception without awareness; mental qualities.

\section{Mental qualities and consciousness}

Properties responsible for the qualitative character of conscious experience are typically referred to as qualia, hereinafter referred to as mental qualities. Some examples of mental qualities are the sweet-sour taste of an orange or the sharp pain of a pinprick. It seems easy to understand what we are talking about when we refer to the taste of an orange or the pain of a pinprick, but it is not easy to give a theoretical account of mental qualities.

There is a rich literature on mental qualities in philosophy that represents several competing theories about them. However, for all this diversity, there are arguably only two approaches to understanding the nature of mental qualities (Rosenthal 2010). One approach identifies mental qualities with the qualitative character of conscious experiences, e.g., the qualitative taste of an orange is whatever it is like to experience the taste an orange consciously. The other approach characterizes mental qualities functionally, relying on the perceptual role they play in the mental economy of an organism, e.g., the qualitative taste of an orange is whatever mental property gives an organism the ability to discriminate it from other tastes. 
The consciousness-based approach is committed to the view that we know about mental qualities by accessing them through consciousness. For example, we identify pains only by way of consciousness (Kripke 1980, p. 149, Putnam 1967). This way of individuating pains from non-pains builds consciousness in, so consciousness is understood to be an intrinsic property of mental qualities.

On the other hand, according to the perceptual-role approach, individual mental qualities are characterized in terms of the relations they bear to other mental qualities in the same family. For example, the taste of an orange is the mental quality that is closer (whatever that means in the context of a particular perceptual-role theory) to the taste of a lemon than to the taste of an avocado in the family of mental qualities of taste. Perceptual-role theories typically characterize these relations functionally at the expense of relying on subjective qualitative character (Rosenthal 1991, Clark 1993). They can do this by, for example, a metric that uses the number of just noticeable differences between individual tastes or colors and then specifies a space of relations using that metric (Clark 1993).

Importantly, perceptual-role theories are not committed to the view that mental qualities always occur consciously, because they do not individuate them by the way they appear subjectively, as is the case with consciousness-based views. According to the perceptual-role approach, the relations that individuate a particular mental quality might be-but not need be-related to consciousness. Of course, this does not mean that they cannot be so connected, but whether they are or not remains an empirical question.

These two approaches-consciousness-based and perceptual-role-can also be adopted in the temporal domain of perceptual experience. On one hand, one could adopt the view that temporal mental qualities (TMQs) such as duration or timing are intrinsically conscious and are individuated by their subjective character. On the other hand, one could adopt the view that TMQs are contingently conscious and individuated functionally.

For a theorist that adopts a consciousness-based approach, the following claim is not only plausible, but a straightforward consequence of the more general thesis about the nature of mental qualities:

(T) If mental state $\mathrm{S}$ features TMQs, then $\mathrm{S}$ is conscious.

Recall that with the consciousness-based approach, if mental state $S$ features any mental qualities, then $S$ is conscious. Therefore, we would need independent reasons to think that this more general claim does not apply to TMQs in particular. 
Edmund Husserl endorsed (T) when he argued that the temporality of consciousness has a protention/primal impression/retention structure (Husserl 1991). If this is right, then every conscious experience has the aforementioned structure. Temporality, therefore, is a structural property of consciousness. One popular interpretation of Husserl's view states that temporality is not only necessary for self-awareness, but is perhaps even constitutive of it (Zahavi 2003, p. 164-70).

A theorist that takes the perceptual-role approach does not have to accept (T). They can stand by a weaker claim, such as: if mental state $S$ features TMQs, then $\mathrm{S}$ may be conscious. Alternatively, if a mental state $\mathrm{S}$ features TMQs, then $\mathrm{S}$ is conscious if condition $\mathrm{C}$ obtains, where $\mathrm{C}$ is some psychological or neural state responsible for conscious experience. Again, for the perceptual-role theorist, whether either of these formulations or some other is true remains an empirical question.

The goal of this paper is to weaken the consciousness-based claim (T). In the following section, I argue that the perceptual-role approach fits better with neuroscientific findings. To that end, I will review some recent experimental work that strongly suggests the existence of a perceptually encoded temporal structure that is used by selective visual attention. In the third section, I consider and dismiss on empirical grounds an objection that disputes my interpretation of empirical work cited in the following section.

\section{Conscious and unconscious temporal structure}

Below I focus on a recent electroencephalography (EEG) study of visual search that measured the N2pc component of an event-related evoked potential (Eimer and Grubert 2014). EEG records electrical potentials using electrodes placed at various sites on the scalp during stimulus presentations. The temporal and spatial profile of the recorded potentials can be used to individuate the neural correlates of functionally individuated psychological processes. For example, the so-called P300 component is an increase in voltage recorded approximately 300 milliseconds after the onset of a perceived visual stimulus in electrodes over the parietal lobe and is typically thought to reflect activation of the neural correlate of object identification and/or conscious experience.

The N2pc component is an electrical negativity that emerges approximately 200 milliseconds after the onset of a stimulus contralaterally to the location of a target stimulus on posterior (occipito-temporal) scalp sites (Luck and Hillyard 1994). Therefore, if a perceiver is asked to visually search for a target object on the left side of their visual field, the N2pc component is likely to occur on the right side of the posterior part of their scalp. N2pc is typically interpreted as a reflection of attentional selection of a visual target during search in the corresponding part of the visual field (Eimer 1996). 
In Eimer and Grubert's 2014 EEG study, participants were presented with two displays in distinct blocks in which the displays were 100,50,20, or 10 milliseconds apart. These displays contained one alphanumerical color-defined target and one alphanumerical distractor. One target-distractor pair was aligned horizontally and the other pair was aligned vertically, with randomized order of alignment. After the displays were presented, participants were asked to indicate with button presses whether the color-defined targets across the two displays belonged to the same alphanumerical category or not. For example, participants pressed one button if the first display featured a red letter and the second display featured a red number. They pressed the other button if both displays featured a blue letter.

The onset of the N2pc component matched the objective time course of the presentations on the computer monitor almost exactly in all the experimental blocks (100, 50, 20, and 10 milliseconds delay between two displays). In other words, the temporal structure of the presentations was encoded and processed by selective attention with a temporal resolution of up to 10 milliseconds. This is striking, because the temporal resolution of conscious visual experience, as measured by temporal order judgments, ${ }^{1}$ is approximately 30 milliseconds (Pöppel 1997, VanRullen, Zoefel, and Ilhan 2014).

Eimer and Grubert's 2014 study shows that selective visual attention can be allocated to onset items at a rate of one per 10 milliseconds. ${ }^{2}$ For example, say we are looking at a two lights, A and B, which are each turning on and off 100 times a second alternatingly. Every 10 milliseconds one light turns on and the other light turns off. Given the temporal resolution of consciousness, in this scenario A and B will appear to be constantly on. Nonetheless, selective attention can switch each time one of the lights comes on. This is because, as the aforementioned experiment demonstrates, selective attention can distinguish onsets at a rate of one every 10 milliseconds.

Selective attention involves visual search and there is no visual search in the aforementioned example with two lights. Therefore, the example involves an intentional simplification. The admittedly idealized scenario is meant to emphasize that the temporal resolution of consciousness is not as high as the temporal resolution of selective attention:

\footnotetext{
${ }^{1}$ Participants make temporal order judgments when they are asked to indicate which of two successive stimuli came after the other. When participants are at chance in their temporal order judgments, it is assumed that they perceive the two successive stimuli as one.

${ }^{2}$ I am very grateful to the authors of this study for stressing to me the importance of it being merely onsets, and not both onsets and offsets to which selective attention is allocated.
} 

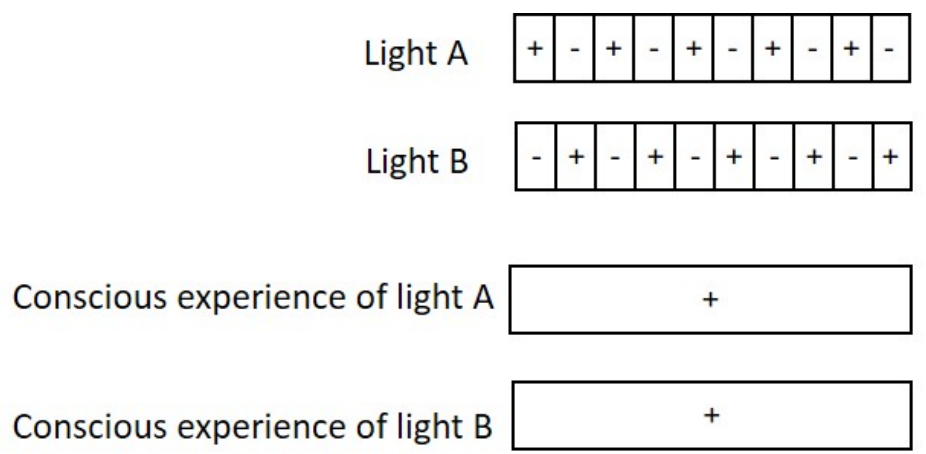

Selective attention switching between A and B \begin{tabular}{|l|l|l|l|l|l|l|l|l|l|}
$\mathrm{A}$ & $\mathrm{B}$ & $\mathrm{A}$ & $\mathrm{B}$ & $\mathrm{A}$ & $\mathrm{B}$ & $\mathrm{A}$ & $\mathrm{B}$ & $\mathrm{A}$ & $\mathrm{B}$ \\
\hline
\end{tabular}

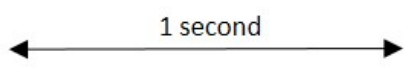

Figure 1. Conscious experience of two lights switching on and off every 10 milliseconds. The plus (+) sign indicates the light being on and the minus sign (-) indicates the light being off.

Figure 1 illustrates how conscious visual experience can present us with two continuous lights, while selective attention switches between them turning on and off. The implications of this finding extend beyond the temporal resolution of perceptual mechanisms. Most importantly, it sheds light on the relationship between perceptually represented temporal structure and conscious experience of that structure, both of which are sometimes thought to be inextricably linked, especially if one accepts (T).

I use the notion of temporal structure to refer to the sum of event boundaries, durations, and the relations between them. The beginning (onset) and end (offset) of a perceivable event flank its duration. Some events happen before others, while some events last longer than others. All of these onsets, offsets, durations, and relations together constitute the temporal structure of the perceivable world. To be successful in a dynamic environment, organisms need to be able to perceive the temporal structure of that environment relatively well.

As numerous studies of time perception demonstrate, the temporal structure of the perceivable environment is not always accurately reflected in human perception (for a review see Grondin, 2010). Certain emotions, for example, make the durations of perceived stimuli appear longer or shorter than they really are (Droit-Volet and Meck 2007) and sounds appear to last longer than lights of equal duration (Wearden 1998). Oddball visual stimuli presented in the middle of a stream of identical visual stimuli appear longer than they ac- 
tually are (Tse et al. 2004). Therefore, there can be a mismatch between the way that parts of the perceivable temporal structure of the world (individual onsets, offsets, durations, etc.) are related to each other and the way they are perceived to be related.

Eimer and Grubert's 2014 EEG study demonstrates that the temporal resolution of the mechanisms of selective visual attention is close to three times that of conscious visual experience. Eimer and Grubert's 2015 behavioral study corroborates this conclusion (Eimer and Grubert 2015). In their study, each participant was presented with two displays of one colored uppercase letter or colored number for 20 milliseconds and asked to report whether the target in the first display was a letter or a number by pressing one of two buttons. This indirectly measured the participants' ability to make temporal order judgments about the two displays, because in order to make that identification, participants had to be able to tell which was first.

The first manipulation was in the stimulus onset asynchrony between the two displays, which was either 10,20 , or 30 milliseconds. The second manipulation was a change in color: on some trials, the color of the target in each display was the same, while in others it was different. Participants saw a letter or number colored red, green, blue, or yellow for 20 milliseconds and then a colored letter or number, either in the same color or some other color, either 10 , 20 , or 20 milliseconds later, and then had to report on the category of the target in the first display.

Results of the aforementioned experiment show that when the displays were separated by 10 milliseconds, participants were at chance in determining the category of the first display. This suggests that they were not able to make explicit temporal order judgments when the two displays were presented at a rate beyond the rate at which their selective attention is able to make distinct temporal orderings. This compliments Gruber and Eimer's 2014 EEG study, which shows that selective visual attention can discriminate visual stimuli 10 milliseconds apart.

This sort of evidence demonstrates that, on average, selective visual attention can be sensitive to more of the relations between the parts of the perceivable temporal structure of the world than can conscious visual experience. As Figure 1 illustrates, the onsets and durations of lights A and B are detected by selective attention and never feature in conscious experience. Therefore, there are relations between onsets, offsets, and durations in the world that are reflected in selective attention, but not in consciousness. ${ }^{3}$

Evidence that parts of the perceivable temporal structure are perceptually encoded without consciousness can be used as an argument against a con-

\footnotetext{
${ }^{3}$ A similar argument based on results of a functional magnetic resonance imagining study of event boundary segmentation in movies is offered in (Klincewicz 2011).
} 
sciousness-based interpretation of (T). According to (T), we would expect that any part of the perceptually encoded temporal structure is inevitably conscious. I have argued that the aforementioned experimental results strongly suggest that this cannot be the case.

A consciousness-based theorist could argue that the EEG evidence presented here merely shows a subpersonal or neural mechanism at work, so it does not challenge (T). In order to provide more definitive evidence against (T), we should have an independent reason to interpret Eimer and Grubert's results as telling us something about perceptual states that are taxonomized folk psychologically. This is what the next section aims to do.

\section{Resisting a consciousness-based interpretation}

The reasons for thinking that Eimer and Grbert's 2014 and 2015 studies are evidence for unconscious perception of temporal structure taxonomized folk psychologically are the following: (1) location of the neural correlates of the N2pc component in the ventral visual stream, which is known to process visual features, such as color, and is connected to consciousness; (2) the relatively late onset of the N2pc component close to the P300 component, which is closely correlated with consciousness; (3) evidence that the N2pc component correlates with the ability to report, which involves processing of high-level perceptual features.

1) The anatomical distribution of the correlates of selective visual attention is telling as it strongly suggests that the mechanisms involved are perceptual and connected to consciousness. Firstly, recall that the N2pc component connected to selective memory is recorded with EEG from posterior areas of the scalp (Luck and Hillyard 1994). These areas of the scalp are directly above the visual cortex. Similarly, magnetoencelographic (MEG) recordings indicate that the $\mathrm{N} 2 \mathrm{pc}$ is generated in areas in the extrastriate occipital-temporal cortex (Hopf et al. 2000), which includes area V4, typically associated with color processing. Moreover, imaging of blood oxygenation levels in the brain using combined functional magnetic resonance/EEG corroborate the cited EEG and MEG recordings, again pointing to the same area, V4, as being particularly involved in selective memory (Hopf et al. 2006).

Secondly, the temporal areas of the visual cortex are all a part of the ventral visual stream, which is tied to conscious visual experience and is distinct from the dorsal visual stream in the parietal areas, which is invariably unconscious, fast, and closely related to action (Goodale and Milner 1992). Neurons in the ventral stream respond selectively to features, such as color or shape, and neurons in the dorsal stream to locations. Availability to consciousness and processing of features are hallmarks of high-level perceptual processing. 
2) The temporal profile of N2pc is also telling because this component occurs relatively late ( 200 milliseconds) after the target visual stimulus, which strongly suggests a substantial amount of prior neural processing. While it remains controversial how long it takes a signal to result in a conscious experience, it has been argued that it could be 240 milliseconds with visual stimuli (Koch 2004, p. 240) and up to 500 milliseconds with tactile stimuli (Libet 2004, p. 76-8, Libet et al. 1979). Recent EEG studies of the temporal profile of visual conscious experience are in line with many previous studies in placing it earlier, namely, at the onset of the P300 component ( 220-300 milliseconds after target) (Rutiku et al. 2015).

Therefore, shortly after the onset of N2pc we typically have a corresponding conscious visual experience, as reflected in the P300 component. This suggests that the final visual percept is ready at the time N2pc occurs right before it is consciously accessed at the time P300 occurs. In line with this idea, some recent theoretical work on the synthesis of global workspace theory of consciousness (Baars 2002) and empirical findings in visual attention both explicitly place the correlate of N2pc right before availability to conscious access (Raffone, Srinivasan, and van Leeuwen 2014, p. 9). This temporal proximity further strengthens the view that selective visual attention operates on representations that include visual features that can be accessed by consciousness.

3) Neither of the previous two reasons is likely to convince a consciousnessbased theorist. This is because anatomical and temporal similarities are vulnerable to the same objection that they aim to answer: they may reflect subpersonal neural mechanisms that cannot be taxonimized folk psychologically. Given this, perhaps the most important reason to resist the consciousnessbased interpretation has to do with the connection that N2pc has to the ability to make verbal reports. This connection suggests that states involved in selective attention have the same role in identification as their conscious counterparts, which constitutes an independent reason to taxonomize them folk psychologically.

We know that ability to report is closely related to conscious experience. For some, verbal reports remain the most common way of operationalizing consciousness in experiments (Seth et al. 2008). This key role for reports arguably comes from the observation that in order to report one has to be, in some way, aware of what one is reporting and, if the report is verbal, form a judgment about it. Conversely, when one is not so aware, one can neither report nor form a judgment.

The same is true of awareness of change: in order to report on the nature of a change, one needs to be able to be aware of it and form an appropriate judgment about it. We have evidence that selective attention reflected in the N2pc component is closely related to the ability to report an identified change (Busch, Fründ, and Herrmann 2010). This suggests involvement of higher- 
cognitive abilities that can access high-level perceptual features in reports of change identification.

As the Busch, Fründ, and Herrmann 2010 study reported, "no change-related positivity or N2pc was observed when the change was only detected or when observers were change blind" (10). Change detection without change identification occurs when one can report that something has changed without being able to report on the nature of the change. For example, reporting that something does not feel the same about the layout of one's bedroom, without being able to tell what it is. Change blindness occurs when no report of change can be made at all.

Therefore, Busch, Fründ, and Herrmann 2010 report that-similar to change blindness-there was no change-related positivity or N2pc component when change was detected, but not identified. When participants could report that they merely sensed something change, they could not report on the features of the change and no N2pc was detected. This strongly suggests that the N2pc component reflects a perceptual mechanism critically tied to how we folk psychologically taxonomize perceptual mental states that may be reported on.

There is also evidence that N2pc can occur without conscious experience that comes from a study of object-substitution masking (Woodman and Luck 2003). The Woodman and Luck study demonstrated that an equivalent N2pc component was present when the identity of a masked target was detected and in trials in which the ability to detect the target was impaired. This suggests that masked targets are accurately identified by the perceptual system, but not passed on to higher-level cognitive systems where they could be used in a report. These results join a number of others that strongly suggest that selective attention/N2pc involves high-level perceptual representations of features that may be used in a report on those features.

While (1)-(3) are not sufficient to resist the consciousness-based interpretation definitively, taken together they make a strong case for the view that the N2pc component is indeed tracking a high-level perceptual mechanism that we should taxonomize folk psychologically. Therefore, it is not likely that the processes reflected by the N2pc are merely neural or subpersonal. It is more reasonable to interpret these processes as a psychological state in the relevant sense and expect these processes to instantiate TMQs.

In general, it may also be worth noting that it is not as controversial as it used to be to think that we can perceive without awareness (Cheesman and Merikle 1986, Merikle, Smilek, and Eastwood 2001, Prinz 2015). There is now a wealth of empirical evidence of perceptually informed performance without awareness: perceptual priming (Marcel 1983), semantic priming (Dehaene et al. 1998), priming during change-blindness (Fernandez-Duque et al. 2003, Fer- 
nandez-Duque and Thornton 2000), blindsight (Kentridge, Heywood, and Weiskrantz 1999, Weiskrantz 1986), relative blindsight induced by metacontrast masking (Lau and Passingham 2006), and blindsight induced with transcranial magnetic stimulation (Ro and Rafal 2006). Given this wealth of evidence in other perceptual domains, it would be surprising if perception of temporal features could not also occur without awareness.

Nonetheless, the debate about the existence of unconscious perception is far from resolved. Ian Phillips has vigorously argued that none of the empirical studies typically brought up as evidence for unconscious perception actually support this claim (Phillips 2015). Other interpretations, Phillips argues, are more compelling. Given this, the interpretation of the evidence from the studies of Eimer and Gruber is, to some extent, hostage to a much larger debate in philosophy about the nature of consciousness.

There also remains the issue of whether the unconscious states putatively involved in effects that seem to support unconscious perception, such as those I brought forward in this paper, involve mental qualities, even if they are taxonomized folk psychologically. Berit Brogaard, for example, argues that classic cases of blindsight should be interpreted as involving thoughts (Brogaard 2011). Ned Block would probably also not accept that unconscious perception involves qualitative mental properties, even though he argues for the existence of unconscious perception (Block 2015, 2011). Geoffrey Lee on the other hand would likely accept that there is unconscious time perception, but probably disagree that unconscious time perception involves TMQs (Lee 2014). Similar distinctions and considerations may apply to the studies of Eimer and Gruber I reported on here, thereby complicating their interpretation and my case against $(\mathrm{T})$.

\section{Conclusion}

In this article, I argued that TMQs can occur without awareness. My argument depends on an interpretation of a recent EEG study, which demonstrates that the temporal resolution of selective visual attention is finer in grain than the temporal resolution of conscious visual experience. This strongly suggests that selective visual attention regularly uses representations of temporal features that never become conscious. The consciousness-based interpretation of (T), which is that if a mental state $S$ features TMQs, then it is conscious, is undermined. In its place, we should accept a perceptual-role version of (T): if mental state $S$ features TMQs, then S may be conscious. Alternatively, if mental state features TMQs, then $S$ is conscious if condition C obtains, where $C$ is some psychological or neural state responsible for conscious experience. 


\section{Acknowledgments}

Work on this paper was financed by the Polish National Science Centre (NCN) SONATA 9 Grant, PSP: K/PBD/000139 under decision UMO-2015/17/D/HS1/ 01705 . The author would like to thank anonymous reviewers of Avant for their constructive comments and suggestions.

\section{Bibliography}

Baars, B. J. 2002. The conscious access hypothesis: origins and recent evidence. Trends in cognitive sciences, 6(1): 47-52.

Block, N. 2015. The Anna Karenina Principle and Skepticism about Unconscious Perception. Philosophy and Phenomenological Research. doi: 10.1111/phpr.12258

Block, N. 2011. The Anna Karenina theory of the unconscious. Neuropsychoanalysis, 13(1): 34-37.

Brogaard, B. 2011. Are there unconscious perceptual processes? Consciousness and cognition, 20(2): 449-463.

Busch, N. A., Fründ, I., Herrmann, Ch. S. 2010. Electrophysiological evidence for different types of change detection and change blindness. Journal of Cognitive Neuroscience, 22(8): 1852-1869.

Cheesman, J., Merikle, P. M. 1986. Distinguishing conscious from unconscious perceptual processes. Canadian Journal of Psychology/Revue canadienne de psychologie, 40(4): 343-367.

Clark, A. 1993. Sensory qualities: Oxford University Press.

Dehaene, S., Naccache, L., Le Clec'H, G., Koechlin, E., Mueller, M., Dehaene-Lambertz, G., van de Moortele, P.-F., Le Bihan, D. 1998. Imaging unconscious semantic priming. Nature, 395(6702): 597-600.

Droit-Volet, S., Meck, W. H. 2007. How emotions colour our perception of time. Trends in Cognitive Sciences, 11(12): 504-513.

Eimer, M. 1996. The N2pc component as an indicator of attentional selectivity. Electroencephalography and clinical neurophysiology, 99(3): 225-234.

Eimer, M., Grubert, A. 2014. Spatial attention can be allocated rapidly and in parallel to new visual objects. Current Biology, 24(2): 193-198.

Eimer, M., Grubert, A. 2015. A dissociation between selective attention and conscious awareness in the representation of temporal order information. Consciousness and cognition, 35: 274-281.

Fernandez-Duque, D., Grossi, G., Thornton, I. M., Neville, H. J. 2003. Representation of change: Separate electrophysiological markers of attention, awareness, and implicit processing. Journal of Cognitive Neuroscience, 15(4): 491-507.

Fernandez-Duque, D., Thornton, I. M. 2000. Change detection without awareness: Do explicit reports underestimate the representation of change in the visual system? Visual Cognition, 7(1-3): 323-344. 
Goodale, M. A., Milner, A. D. 1992. Separate visual pathways for perception and action. Trends in neurosciences, 15(1): 20-25.

Grondin, S. 2010. Timing and time perception: a review of recent behavioral and neuroscience findings and theoretical directions. Attention, Perception, \& Psychophysics, 72(3): 561-582.

Hopf, J.-M., Luck, S. J., Boelmans, K., Schoenfeld, M. A., Boehler, C. N., Rieger, J., Heinze, H.-J. 2006. The neural site of attention matches the spatial scale of perception. The Journal of Neuroscience, 26(13): 3532-3540.

Hopf, J.-M., Luck, S. J., Girelli, M., Hagner, T., Mangun, G. R., Scheich, H., Heinze, H.-J. 2000. Neural sources of focused attention in visual search. Cerebral Cortex, 10(12): 1233-1241.

Husserl, E. G. 1991. On the Phenomenology of the Consciousness of Internal Time (18931917). Translated by John Barnett Brough: Dordrecht: Kluwer.

Kentridge, R. W., Heywood, Ch. A., Weiskrantz, L. 1999. Attention without awareness in blindsight. Proceedings of the Royal Society of London. Series B: Biological Sciences, 266(1430): 1805-1811.

Klincewicz, M. 2011. Quality space model of temporal perception. In Multidisciplinary Aspects of Time and Time Perception, 230-245. Springer.

Koch, Ch. 2004. The quest for consciousness: a neuroscientific approach. Colorado: Roberts \& Company Publishers.

Kripke, S. A. 1980. Naming and necessity. Cambridge, Mass.: Harvard University Press.

Lau, H. C., Passingham, R. E. 2006. Relative blindsight in normal observers and the neural correlate of visual consciousness. Proceedings of the National Academy of Sciences, 103(49): 18763-18768.

Lee, G. 2014. Temporal Experience and the Temporal Structure of Experience. Philosopher's Imprint, 14(3).

Libet, B. 2004. Mind time: The temporal factor in consciousness. Cambridge, Mass.: Harvard Univ Press.

Libet, B., Wright, E. W., Feinstein Jr., B., Pearl, D. K. 1979. Subjective referral of the timing for a conscious sensory experience: a functional role for the somatosensory specific projection system in man. Brain, 102(1): 193-224.

Luck, S. J., Hillyard, S. A. 1994. Spatial filtering during visual search: evidence from human electrophysiology. Journal of Experimental Psychology: Human Perception and Performance, 20(5): 1000-1014.

Marcel, A. J. 1983. Conscious and unconscious perception: An approach to the relations between phenomenal experience and perceptual processes. Cognitive psychology, 15(2): 238-300.

Merikle, P. M., Smilek, D., Eastwood, J. D. 2001. Perception without awareness: Perspectives from cognitive psychology. Cognition, 79(1): 115-134.

Phillips, I. 2015. Consciousness and criterion: on Block's case for unconscious seeing. Philosophy and Phenomenological Research. doi: 10.1111/phpr.12224

Pöppel, E. 1997. A hierarchical model of temporal perception. Trends in Cognitive Sciences, 1(2): 56-61. 
Prinz, J. J. 2015. Unconscious Perception. The Oxford Handbook of the Philosophy of Perception: 371.

Putnam, H. 1967. Psychological predicates. Art, mind, and religion, 1: 37-48.

Raffone, A., Srinivasan, N., van Leeuwen, C. 2014. The interplay of attention and consciousness in visual search, attentional blink and working memory consolidation. Philosophical Transactions of the Royal Society of London B: Biological Sciences, 369(1641): 20130215.

Ro, T., Rafal, R. 2006. Visual restoration in cortical blindness: insights from natural and TMS-induced blindsight. Neuropsychological rehabilitation, 16(4): 377-396.

Rosenthal, D. M. 1991. The independence of consciousness and sensory quality. In Consciousness (Philosophical Issues 1), edited by Enrique Villanueva, 15-36. Atascadero, CA: Ridgeview Publishing.

Rosenthal, D. M. 2010. How to think about mental qualities. Philosophical Issues, 20(1): 368-393.

Rutiku, R., Martin, M., Bachmann, T., Aru, J. 2015. Does the P300 reflect conscious perception or its consequences? Neuroscience, 298: 180-189.

Seth, A. K., Dienes, Z., Cleeremans, A., Overgaard, M., Pessoa, L. 2008. Measuring consciousness: relating behavioural and neurophysiological approaches. Trends in Cognitive Sciences, 12(8): 314-321.

Tse, P. U., Intriligator, J., Rivest, J., Cavanagh, P. 2004. Attention and the subjective expansion of time. Perception \& Psychophysics, 66(7): 1171-1189.

VanRullen, R., Zoefel, B., Ilhan, B. 2014. On the cyclic nature of perception in vision versus audition. Philosophical Transactions of the Royal Society B: Biological Sciences, 369(1641): 20130214.

Wearden, J., Edwards, H., Fakhri, M., Percival, A. 1998. Why "Sounds Are Judged Longer Than Lights”: Application of a Model of the Internal Clock in Humans. The Quarterly Journal of Experimental Psychology B, 51(2): 97-120.

Weiskrantz, L. 1986. Blindsight: A case study and implications. Oxford: Oxford University Press.

Woodman, G. F., Luck, S. J. 2003. Dissociations among attention, perception, and awareness during object-substitution masking. Psychological Science, 14(6): 605-611.

Zahavi, D. 2003. Inner time-consciousness and pre-reflective self-awareness. In The New Husserl: A Critical Reader, edited by Donn Welton, 157-180. Bloomington: Indiana University Press. 